\title{
Highly Scalable Compression Method for Super Resolution Multi Spectral Images
}

\author{
V. Bhagya Raju ${ }^{1}$, Dr. K. Jaya Shankar ${ }^{2}$, Dr. C. D. Naidu ${ }^{3}$ \\ Research Scholar ${ }^{1}$, Professor \& Head of ECE ${ }^{2}$,Professor \& Principal ${ }^{3}$ \\ JNTU Hyderabad ${ }^{l}$ \\ Vasavi College of Engineering ${ }^{2}$ \\ VNR VJIET, Hyderabad
}

\begin{abstract}
This paper provides a new technique for lossy multispectral images compression at very high data rates. Image compression is becoming more and more important, as new multispectral instruments are going to generate very high data rates due to the increased spatial and spectral resolutions. The Lossless compression does not provide a sufficient degree of data volume reduction to meet the bandwidth requirements of the downlink channel, so a lossy approach is the only possible solution. Multispectral data exhibits a large spectral correlation. Many techniques have been proposed for multispectral image data. Mainly in all the compression techniques to minimize the intra-band and inter-band correlation, some of them do in a single step and others do in a two-step transformation followed by an encoding scheme to eliminate the statistical redundancy or to give a desired bit-rate at low compression ratios based on the wavelet transform, where the spatial and the spectral redundancy are removed simultaneously. At higher compression ratios, a high quality wavelet based method in the spatial dimensions combined with the Improved Set Partitioning Hierarchical Trees (ISPHIT) in the spectral dimension gives the best results.
\end{abstract}

Keywords: Multi spectral images, Super resolution, ISPHIT, LIBT, LIST

\section{Introduction}

Multispectral image compression algorithms can be roughly categorized by how they exploit the redundancies along the spatial and spectral dimensions. A hyper/multispectral imaging system splits the light spectrum into more than three frequency bands (dozens to hundreds) and records each of the images separately as a set of monochrome images. This type of technique increases the number of acquisition channels in the visible spectrum and expands channel acquisition to the light that is outside the sensitivity of the human eye. Such systems offer several advantages over conventional RGB imaging and have, therefore, attracted increasing interest in the past few years. However, multispectral uncompressed images, in which single image band may occupy hundreds of megabytes, often require high capacity storage. Compression is thus necessary to facilitate both the storage and the transmission of multispectral images. The simplest method for compressing multispectral data is to decompose the multispectral image into a set of monochrome images, and then to separately compress each image using conventional image compression methods. Other multispectral compression techniques concentrate solely on the spectral redundancy. However, the best compression methods exploit redundancies in both the spatial and spectral dimensions. As with monochrome image compression, multispectral image compression algorithms fall into two general categories: lossless and lossy. In lossless compression schemes, the decoded image is identical to the original. This gives perfect fidelity but limits the achievable compression ratio. For many applications, the required compression ratio is larger than can be achieved with lossless compression, so lossy algorithms are used. Lossy algorithms typically obtain much higher compression ratios but introduce distortions in the decompressed image. Lossy compression algorithms attempt to introduce errors in such a way as to minimize the degradation in output image quality for a given compression ratio. In fact, the rate distortion curve gives the minimum bitrates (and hence maximum compression) required to achieve a given distortion. If the allowed distortion is taken to be zero, the resulting maximum compression is the limit for lossless coding. The limit obtained from the theoretical rate distortion curve can be useful for evaluating the effectiveness of a given algorithm. While the bound is usually computed with respect to mean squared error (MSE) distortion, MSE is not a good measure of quality in all applications. However, usages of multispectral data are dependent mostly on rich spectral information contained in such images. So a practical algorithm for multispectral data should preserve spectral characteristics of the data while working in a lossy manner and maximize the gain.

The two-dimensional image coding algorithms attempt to transform the image data so that the transformed data samples are largely uncorrelated. The samples can then be quantized independently and entropy coded. At the decoder, the quantized samples are recovered and inverse transformed to produce the 
reconstructed image. The multi spectral images are shown in Fig 1. The recent approaches to lossless multispectral compression of high spectral resolution images include transform-based, prediction based and vector quantization methods [1] and image data based on prediction tree was given by Memon et al. [2]. The 3D CALIC (context-based adaptive lossless image codec) [2] was also proposed. Zhang and Yan [3] changed the definition of the four neighbourhood model of prediction tree and gave side neighbourhood minimum absolute weight prediction tree. Later, $\mathrm{Wu}$ and $\mathrm{He}$ [4] made an improvement on the method of prediction tree by setting up adaptive predictor aiming at multispectral images' statistic spectral redundancy. The prediction estimate uses two pixels in the previous bands in the same spatial position as the current pixel. By comparison, Rizzo et al. [5] gave adaptive least-squares optimized prediction technique called spectrum oriented least squares (SLSQ). The prediction coefficients can be computed using an offline procedure on training data. The remainder of this paper is organized as follows. Section II briefly describes the Super Resolution Image. Section III introduces a Discrete Wavelet Decomposition. Section IV will discuss the ISPIHT Algorithm based compression technique for Multispectral image compression. Section V presents performance Evaluation and qualitative and quantitative experimental results. Section VI concludes with some remarks.
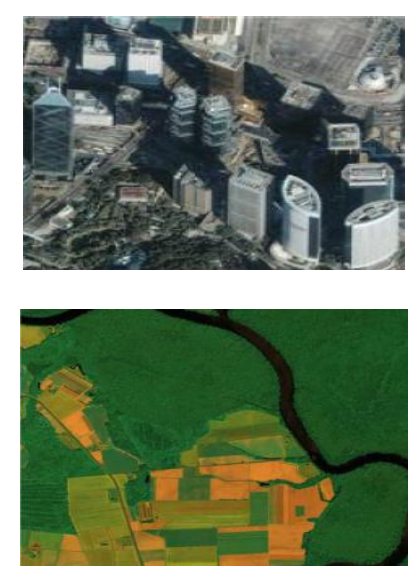

Fig 1. Remotely sensed multispectral image
a) Hong Kong city
b) AVIRIS Image

\section{Image Super- Resolution}

An image super-resolution [6], [7] is obtained using a generic image gradient profile prior, which is a novel parametric approach for describing the shape and the sharpness of the image learned from a large number of natural images.

Super-resolution is to estimate a hi-resolution (HR) image from a low-resolution (LR) input. There are mainly three categories of approach for this problem: interpolation based methods, reconstruction based methods, and learning based methods. The interpolation based methods are simple but tend to blur the high frequency details. The reconstruction based methods enforce a reconstruction constraint which requires that the smoothed and down-sampled version of the HR image should be close to the LR image. The learning based methods "hallucinate" high frequency details from a training set of HR/LR image pairs. The learning based approach highly relies on the similarity between the training set and the test set.

To design a good image super-resolution algorithm, the essential issue is how to apply a good prior or constraint on the HR image because of the ill-posedness of the image super-resolution. Generic smoothness prior and edge smoothness prior are two widely used priors.

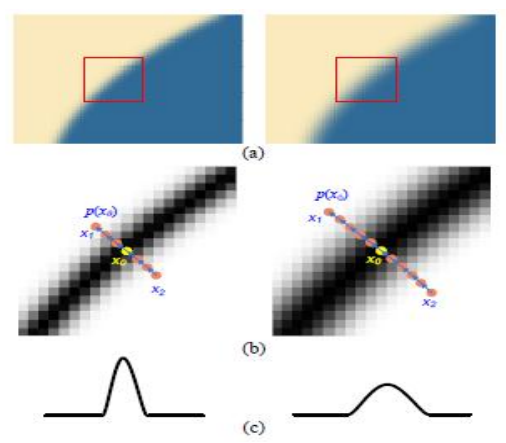

Fig 2. Gradient Profile.

(a)Two edges with different sharpness. 
(b)Gradient maps (normalized and inverted magnitude) of two rectangular regions in (a). $\mathrm{p}(\mathrm{x} 0$ ) is a gradient profile passing through the edge pixel (zero crossing pixel) $\mathrm{x} 0$, by tracing along gradient directions (two sides) pixel by pixel until the gradient magnitude does not decrease at $\mathrm{x} 1$ and $\mathrm{x} 2$.

(c) 1D curves of two gradient profiles.

The gradient profile is a 1-D profile along the gradient direction of the zero-crossing pixel in the image. The gradient profile prior is a parametric distribution describing the shape and the sharpness of the gradient profiles in natural image. One of our observations is that the shape statistics of the gradient profiles in natural image is quite stable and invariant to the image resolution. With this stable statistics, we can learn the statistical relationship of the sharpness of the gradient profile between the HR image and the LR image. Using the learned gradient profile prior and relationship, we are able to provide a constraint on the gradient field of the HR image. Combining with the reconstruction constraint, we can recover a hi-quality HR image.

\section{A. Gradient Profile Prior}

Previous natural image statistics characterizes the marginal distribution of the image gradients over the whole image. The spatial information is discarded. Instead, we study the image gradients along local image structures and the statistical dependency of the image gradients between the HR image and the LR image.

Gradient profile and its sharpness

Denote the image gradient as $\nabla \mathrm{I}=\mathrm{m} . \mathrm{N}$, where ' $\mathrm{m}$ ' is the gradient magnitude and ' $\mathrm{N}$ ' is the gradient direction. In the gradient field, we denote the zero crossing pixel which is the local maximum on its gradient direction as edge pixel Fig 2(a) is two image blocks containing two edges with different sharpness. Fig 2(b) are corresponding gradient (magnitude) maps. The pixel x0 in Fig 2(b) is a zero crossing or edge pixel. Starting from $\mathrm{x} 0$, we trace a path along the gradient directions (two-sides) pixel by pixel until the gradient magnitude does not decrease anymore.

The 1-D path $\mathrm{p}(\mathrm{x} 0)$ is called as gradient profile. Fig 2. (c) is 1D curves of two gradient profiles. We measure the sharpness of the gradient profile using the square root of the variance (second moment):

$$
\sigma\left(p\left(x_{o}\right)\right)=\sqrt{\sum_{x \in p\left(x_{o}\right)} m^{o}(x) d^{2}\left(x, x_{o}\right)}
$$

Where $m^{o}(x)=\frac{m(x)}{\sum_{x \in p\left(x_{o}\right)} m(s)}$ and $\mathrm{d}(\mathrm{x}, \mathrm{x} 0)$ is the curve length of the gradient profile between $\mathrm{x}$ and $\mathrm{x} 0$. Sharper the image gradient profile, the smaller the variance ' $\sigma$ ' is and we call this variance as the profile sharpness.

Profile sharpness estimation

Individually estimating the sharpness for each gradient profile is not robust due to the noise. To have a better estimation, we apply a global optimization to enforce the consistency of neighbouring profiles as follows. First, we construct a graph on all edge pixels. The graph node is the edge pixel and the graph edge is the connection between two neighbouring edge pixels within a pre-defined distance (5 pixels). The edge weight $\mathrm{w}_{\mathrm{ij}}$ for each clique of two connected nodes $i$ and $\mathrm{j}$ is defined as,

$W_{i, j}=\exp \left(-\varsigma_{1} \cdot\left|\nabla u_{i}-\nabla u_{j}\right|^{2}-\varsigma_{2} \cdot d(i, j)^{2}\right)$

Where the first term in the exponent is the gradient similarity and the second term is Euclidean distance between $i$ and $j$. For each node $i$, we individually estimate its sharpness $\sigma_{\mathrm{i}}$. Then, we minimize the following energy to estimate the sharpness of all edge pixels:

$$
E\left(\left\{\sigma_{i}\right\}\right)=\sum_{i}\left[\left(\sigma_{i}-\hat{\sigma}_{j}\right)^{2}+\gamma \cdot \sum_{j \in N(i)} W_{i, j}\left(\sigma_{i}-\sigma_{j}\right)^{2}\right]
$$

(3) where N(i) are neighbouring zeros of the node

i. This energy can be effectively minimized because it is an Gaussian MRF model, in which $\gamma=5, \zeta_{1}=0.15$, and $\zeta_{2}=0.08$ in our implementation.

\section{B.Distribution Gradient profile prior}

The regularity of the gradient profiles in natural image can be investigated by distribution of the gradient profile. The general exponential family distribution, i.e. Generalized Gaussian Distribution (GGD) is defined as,

$$
g(x ; \sigma, \lambda)=\frac{\lambda \alpha(\lambda)}{2 \sigma(1 / \lambda)} \exp \left\{1-\left[\alpha(\lambda)\left|\frac{x}{\sigma}\right|\right]^{\lambda}\right\}
$$

the scaling factor which makes the second moment of GGD equal to $\sigma^{2}$. Therefore, $\sigma$ can be directly estimated using the second moment of the profile. $\lambda$ is the shape parameter which controls the overall shape of the distribution. Using Kullback - Leibler (KL) divergence to measure the fitting error, we estimate the optimal $\lambda$ by 
$\lambda=\arg \min _{\lambda}\left\{\sum_{p \in \Omega} K L\left(p, g\left(\sigma_{p}, \lambda\right)\right)\right\}$

where $\sigma_{p}$ is the variance of $p$, which is one profile in the set $\Omega$.

Compute the average KL divergences on four profile sets $\Omega_{1}, \Omega_{2}, \Omega_{3,}, \Omega_{4, \text { by }}$ varying the shape parameter $\lambda$. As we can see, the optimal shape parameter is about 1.6 for all down-sampling factors. The shape parameter $\chi^{2}$ is stable across different resolutions, which means that the gradient profile distribution is resolution independent in natural image.

We use Pearson's $\chi^{2}$ hypothesis-tests to measure the goodness of our fitted distributions. The $\chi^{2}$ hypothesistest for a gradient profile $\mathrm{p}(\mathrm{x} 0)$ is defined as

$$
\begin{aligned}
& \chi^{2}(p)=\sum_{x \in p\left(x_{o}\right)} \frac{[m(x)-E(x)]^{2}}{E(x)} \\
& E(x)=\frac{g\left(d\left(x, x_{o}\right)\right)}{=\sum_{s \in p\left(x_{o}\right)} g\left(d\left(x, x_{o}\right)\right)} \sum_{s \in p\left(x_{o}\right)} m(s)
\end{aligned}
$$

$\mathrm{n}-1$ ( $\mathrm{n}$ is the number of pixels in $\mathrm{p})$, if $\chi^{2}(\mathrm{p})<\chi^{2}(\mathrm{k}, \mathrm{n}-1)$ the hypothesis that the gradient profile follows the fitted gradient profile prior cannot be rejected.

\section{Selection of Representative Spectral Bands}

In the proposed method, the representative spectral bands which contain maximum information are selected. To select these representative bands, the entropy of each band, and spectral correlation between the adjacent bands are calculated, and then the bands having maximum entropy and de-correlation are selected. These representative bands are then transformed by using discrete wavelet transform and encoded using Improved SPIHT algorithm.

In this work, we use DWT for multispectral image compression. We make an assumption that spectral correlation exists in wavelet coefficients of multispectral image. This scheme exploits the spatial and spectral correlation in the biorthogonal subclasses of multispectral image. The proposed scheme consists of two steps. First, all bands of multispectral image are analyzed independently using two-dimensional Discrete Wavelet Transform. Then, the wavelet coefficients are quantized with Improved SPHIT.

\section{A. Subband Coding}

In subband coding, an image is decomposed into a set of band limited components, called sub bands, which can be reassembled to reconstruct the original image without error. Fig 3 shows the components of twoband subband coding and decoding system. Since the bandwidth of the resulting sub bands $y_{0}(n)$ or $y_{1}(n)$ is smaller than the original signal $\mathrm{x}(\mathrm{n})$, the sub bands can be down sampled without loss of information. Reconstruction of the original signal is accomplished by up sampling, filtering, and summing the individual sub bands.

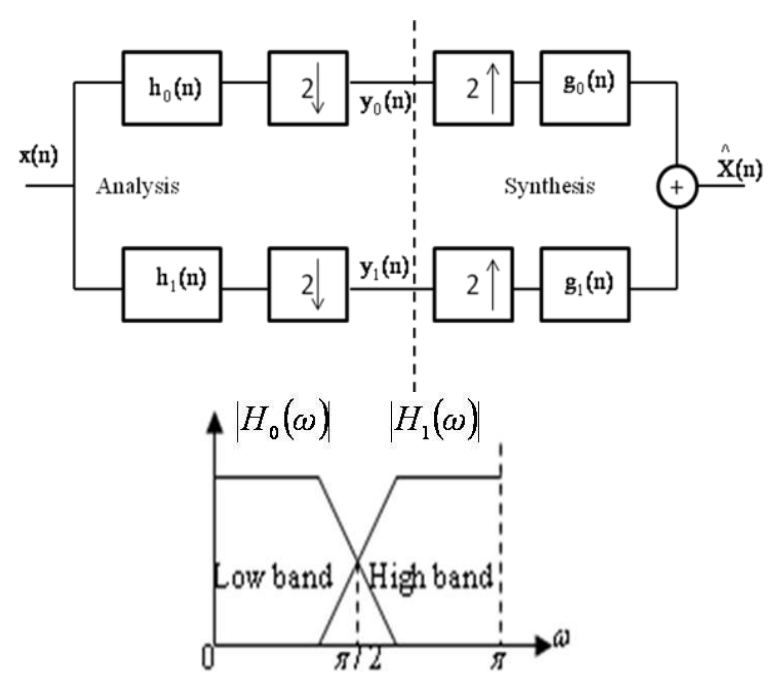

Fig 3. Two-band filter bank for one-dimension subband coding and decoding. 
According to the Z-transform and its sampling theorem, we can express the output as

$$
\begin{aligned}
\hat{X}(z)= & \frac{1}{2}\left[H_{0}(z) G_{0}(z)+H_{1}(z) G_{1}(z)\right] X(z) \\
& +\frac{1}{2}\left[H_{0}(-z) G_{0}(z)+H_{1}(-z) G_{1}(z)\right] X(-z)
\end{aligned}
$$

Where the second component which contains the $-\mathrm{z}$ dependence represents the aliasing introduced by the down sampling-up sampling process.

For error-free reconstruction of the input, that is, $\hat{X}(z)=X(z)$ we impose the following conditions:

$H_{0}(-z) G_{0}(z)+H_{1}(-z) G_{1}(z)=0$

$H_{0}(z) G_{0}(z)+H_{1}(z) G_{1}(z)=2$

Eq. (9) reveals that the analysis and synthesis filter are cross-modulated. For finite impulse response (FIR) filters and ignoring the delay, we can get

$$
\begin{aligned}
& g_{0}(n)=(-1)^{n} h_{1}(n) \\
& g_{1}(n)=(-1)^{n+1} h_{0}(n)
\end{aligned}
$$

Thus, FIR synthesis filters are cross-modulated copies of the analysis filters with one (and only one) being sign reversed.

Eq. (9) can also be used to demonstrate the biorthogonality of the analysis and synthesis filters. That is

$$
\begin{aligned}
& <g_{0}(k), h_{0}(2 n-k)>=\delta(n) ; \\
& <g_{1}(k), h_{1}(2 n-k)>=\delta(n) ; \\
& <g_{0}(k), h_{1}(2 n-k)>=0 ; \\
& <g_{1}(k), h_{0}(2 n-k)>=0 ; \\
\left\langle h_{i}(2 n-k), g_{j}(k)\right\rangle= & \delta(i-j) \delta(n), \quad i, j=\{0,1\}
\end{aligned}
$$

Filter banks satisfying this condition are called biorthogonal. Moreover, the analysis and synthesis filter impulse responses of all two-band, real-coefficient; perfect reconstruction filter banks are subject to the biorthogonality constraint.

One solution that satisfies the biorthogonality requirement of Eq. (12) and used in the development of the fast wavelet transform are called orthonormal. It require

$$
\left\langle g_{i}(n), g_{j}(n+2 m)\right\rangle=\delta(i-j) \delta(m), \quad i, j=\{0,1\}
$$

which defines orthonormality for perfect reconstruction filter banks. The relationship of the four filter is

$$
\begin{aligned}
& g_{1}(n)=(-1)^{n} g_{0}(2 K-1-n) \\
& h_{i}(n)=g_{i}(2 K-1-n), \quad i=\{0,1\}
\end{aligned}
$$

From Eq. (15) $2 \mathrm{~K}$ denotes the number of coefficients in each filter. As can be seen, $\mathrm{g}_{1}(\mathrm{n})$ is related to $\mathrm{g}_{0}(\mathrm{n})$ and both $\mathrm{h}_{0}(\mathrm{n})$ and $\mathrm{h}_{1}(\mathrm{n})$ are time-reversed versions of $\mathrm{g}_{1}(\mathrm{n})$ is related to $\mathrm{g}_{0}(\mathrm{n})$, respectively.

\section{B. Multi Resolution Analysis}

In multiresolution analysis, a scaling function is used to create a series of approximations of a signal. A wavelet function is used to encode the difference in information between adjacent approximations. A signal $\mathrm{f}(\mathrm{x})$ can be analyzed as a linear combination of expansion functions

$$
f(x)=\sum_{k} \alpha_{k} \phi_{k}(x)
$$

Where the $\alpha_{\mathrm{k}}$ is real-valued expansion coefficients and the $\emptyset_{\mathrm{k}}(\mathrm{x})$ are real-valued expansion functions. If the expansion is unique, the $\emptyset_{k}(x)^{\phi_{k}(x)}$ are called basis functions. The function space of the expansion set $\left(\emptyset_{\mathrm{k}}\right.$ $(\mathrm{x}))$ is

$$
V=\operatorname{span}_{k}\left\{\phi_{k}(x)\right\}
$$

And $f(x) \in V$ means that $f(x)$ is in the span of $\left(\varnothing_{k}(x)\right)$ and can be written in the form of Eq. (17). The coefficients $\alpha_{\mathrm{k}}$ are computed by taking the integral inner products of the dual $\tilde{\phi}_{k}(x)$ 's and function $\mathrm{f}(\mathrm{x})$. That is $\alpha_{k}=\left\langle\tilde{\phi}_{k}(x), f(x)\right\rangle=\int \tilde{\phi}_{k}^{*}(x) f(x) d x$

If $\left(\varnothing_{\mathrm{j}, \mathrm{k}}(\mathrm{x})\right)$ is an orthonormal basis for $\mathrm{V}$, then $\phi_{k}(x)=\tilde{\phi}_{k}(x)$. If $\left(\emptyset_{\mathrm{k}}(\mathrm{x})\right)$ are not orthonormal but is an orthogonal basis for $\mathrm{V}$, then the basis functions and their duals are called biorthogonal. That is 
$\left\langle\phi_{j}(x), \tilde{\phi}_{k}(x)\right\rangle=\delta_{j k}= \begin{cases}0 & , j \neq k \\ 1 & , j=k\end{cases}$

Now consider the set of expansion functions $\left(\varnothing_{\mathrm{j}, \mathrm{k}}(\mathrm{x})\right)$ composed of integer translations and binary scaling of the real, square-integral function $\emptyset(\mathrm{x})$ where

$\phi_{j, k}(x)=2^{j / 2} \phi\left(2^{j} x-k\right)$

for $k \in Z$ and $\emptyset(x) \in L^{2}(R)$. Because the shape of $\emptyset_{j, k}(x)$ changes with $j, \emptyset(x)$ is called a scaling function. We denote the subspace spanned over $\mathrm{k}$ for any $\mathrm{j}$ as

$V_{j}=\operatorname{span}_{k}\left\{\phi_{j, k}(x)\right\}$

The scaling function has four fundamental requirements of multiresolution analysis:

1. The scaling function is orthogonal to its integer translates.

2. The subspaces spanned by the scaling function at low scales are nested within those spanned at higher scales. That is

$V_{-\infty} \subset \cdots \subset V_{-1} \subset V_{0} \subset V_{1} \subset V_{2} \subset \cdots \subset V_{\infty}$.

1. The only function that is common to all $V_{j}$

is $f(x)=0$. That is

$V_{-\infty}=\{0\}$.

2. Any function can be represented with arbitrary precision. That is,

$V_{\infty}=\left\{L^{2}(\mathbf{R})\right\}$

The expansion functions of any subspace can be built from double-resolution copies of themselves. That is,

$\phi(x)=\sum_{n} h_{\phi}(n) \sqrt{2} \phi(2 x-n)$

where the $h_{\varnothing}(n)$ coefficients are called scaling function coefficients. Given a scaling function that meets the multiresolution requirements, we can define a wavelet function $\psi(\mathrm{x})$ that spans the difference between any two adjacent scaling subspaces, $\mathrm{V}_{\mathrm{j}}$ and $\mathrm{V}_{\mathrm{j}+1}$. We can define the set $\left(\psi_{\mathrm{j}, \mathrm{k}}(\mathrm{x})\right)$ of wavelets

$\psi_{j, k}(x)=2^{j / 2} \psi\left(2^{j} x-k\right)$

for all $k \in \mathbf{Z}$ that spans the space $W_{j}$ where

$W_{j}=\underset{k}{\operatorname{span}}\left\{\psi_{j, k}(x)\right\}$.

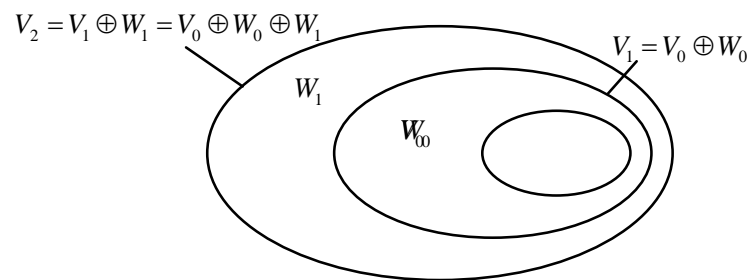

Fig 4. The relationship between scaling and wavelet function spaces.

$V_{j+1}=V_{j} \oplus W_{j}$.

$L^{2}(\mathbf{R})=V_{0} \oplus W_{0} \oplus W_{1} \oplus W_{2} \oplus \cdots$

$L^{2}(\mathbf{R})=\cdots \oplus W_{-2} \oplus W_{-1} \oplus W_{0} \oplus W_{1} \oplus W_{2} \oplus \cdots$

Or Even

Similar to the scaling function, the wavelet function can be expressed as a weighted sum of shifted, doubleresolution scaling functions. That is,

$\psi(x)=\sum_{n} h_{\psi}(n) \sqrt{2} \phi(2 x-n)$

where the $h_{\psi}(n)$ are called the wavelet function coefficients. It can be shown that $h_{\psi}(n)$ is related to $h_{\varnothing}$ (n) by

$h_{\psi}(n)=(-1)^{n} h_{\phi}(1-n)$.

C. Discrete Wavelet Transform 
We begin by defining the wavelet series expansion of function $\mathrm{f}(\mathrm{x}) \mathrm{CL} \mathrm{L}^{2}(\mathrm{R})$ relative to wavelet $\psi(\mathrm{x})$ and scaling function $\varnothing(\mathrm{x})$. We can write

$$
f(x)=\sum_{k} c_{j_{0}}(k) \phi_{j_{0}, k}(x)+\sum_{j=j_{0}}^{\infty} \sum_{k} d_{j}(k) \psi_{j, k}(x)
$$

Where $\mathrm{j}_{0}$ is an arbitrary starting scale and the $\mathrm{c}_{\mathrm{jo}}(\mathrm{k})$ are normally called the approximation or scaling coefficients, the $\mathrm{d}_{\mathrm{j}}(\mathrm{k})$ are called the detail or wavelet coefficients. The expansion coefficients are calculated as

$$
\begin{aligned}
& c_{j_{0}}(k)=\left\langle f(x), \tilde{\phi}_{j_{0}, k}(x)\right\rangle=\int f(x) \tilde{\phi}_{j_{0}, k}(x) d x \\
& d_{j}(k)=\left\langle f(x), \tilde{\psi}_{j, k}(x)\right\rangle=\int f(x) \tilde{\psi}_{j, k}(x) d x
\end{aligned}
$$

If the function is being expanded is a sequence of numbers, like samples of a continuous function $\mathrm{f}(\mathrm{x})$. The resulting coefficients are called the discrete wavelet transform (DWT) of $\mathrm{f}(\mathrm{x})$. Then the series expansion defined in Eqs. (34) and (35) becomes the DWT transform pair

$$
\begin{aligned}
& W_{\phi}\left(j_{0}, k\right)=\frac{1}{\sqrt{M}} \sum_{x=0}^{M-1} f(x) \tilde{\phi}_{j_{0}, k}(x) \\
& W_{\psi}(j, k)=\frac{1}{\sqrt{M}} \sum_{x=0}^{M-1} f(x) \tilde{\psi}_{j, k}(x) \\
& f(x)=\frac{1}{\sqrt{M}} \sum_{k} W_{\phi}\left(j_{0}, k\right) \phi_{j 0, k}(x) \\
& \text { for } j \geq j_{0} \text { and } \\
& +\frac{1}{\sqrt{M}} \sum_{j=j_{0}}^{\infty} \sum_{k} W_{\psi}(j, k) \psi_{j, k}(x)
\end{aligned}
$$
where $f(x), \phi_{j_{0}, k}(x)$, and

$\psi_{j, k}(x)$ are functions of discrete variable $\mathrm{x}=0,1,2, \ldots, \mathrm{M}-1$.

D. Wavelet Transforms in Two Dimensions

In two dimensions, a two-dimensional scaling function, $\varnothing(x, y)$ and three two-dimensional wavelet $\psi_{(\mathrm{x}, \mathrm{y})}^{\mathrm{H}}, \psi^{\mathrm{V}}{ }_{(\mathrm{x}, \mathrm{y})}$ and $\psi_{(\mathrm{x}, \mathrm{y})}^{\mathrm{D}}$, are required. Each is the product of a one-dimensional scaling function $\phi$ and corresponding wavelet $\psi$.

$\phi(x, y)=\phi(x) \phi(y)$

$\psi^{H}(x, y)=\psi(x) \phi(y)$

$\psi^{V}(x, y)=\phi(y) \psi(x)$

$\psi^{D}(x, y)=\psi(x) \psi(y)$

Where $\psi^{\mathrm{H}}$ measures variations along columns (like horizontal edges), $\psi^{\mathrm{V}}$ responds to variations along rows (like vertical edges), and $\psi^{\mathrm{D}}$ corresponds to variations along diagonals.

Like the one-dimensional discrete wavelet transform, the two-dimensional DWT can be implemented using digital filters and down samplers. With separable two-dimensional scaling and wavelet functions, we simply take the one-dimensional FWT of the rows of $\mathrm{f}(\mathrm{x}, \mathrm{y})$, followed by the one-dimensional FWT of the resulting columns.

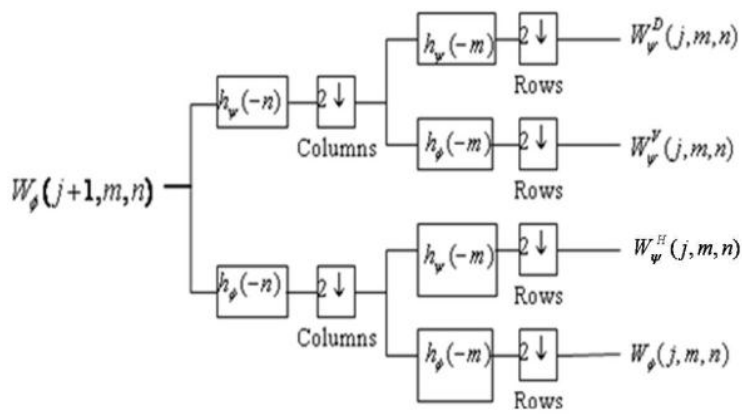

Fig 5. The two-dimensional FWT - the analysis filter bank 


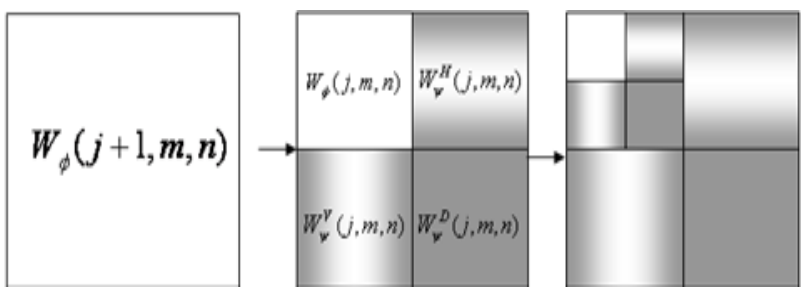

Fig 6. Two-Scale of Two -Dimensional Decomposition

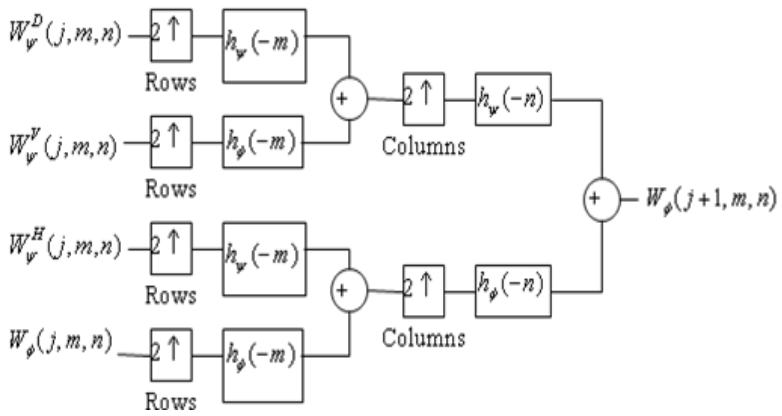

Fig 7. The two-dimensional DWT - The synthesis filter bank

The single-scale filter bank of Fig 6 can be "iterated" by tying the approximation output to the input of another filter bank to produce a arbitrary scale transform. As in the one-dimensional case, image $f(x, y)$ is used as the first scale input, and output four quarter-size sub images $-\mathrm{W}_{\varnothing} \mathrm{W} \psi^{\mathrm{H}}, \mathrm{W} \psi^{\mathrm{V},}$ and $\mathrm{W} \psi^{\mathrm{D}}$. These sub images are shown in the middle of Fig 6. Two iterations of the filtering process produce the two-scale decomposition at the right of Fig 6. The synthesis filter bank that reverses the process described above is shown in Fig 7.

\section{Improved Set Portioning In Hierarchical Trees (ISPIHT)}

ISPIHT is the wavelet based image compression method which provides the highest image quality [8]. The improved SPIHT algorithm mainly makes the following changes. SPIHT codes four coefficients and then shifts to the next four ones and views the four coefficients as a block. The maximum of them regarded as the compared threshold will decrease number of comparison, which is related to the distribution of coefficient matrix. Even more, when the maximum in the block is smaller than the current threshold or equal to it, the block will be coded with only one bit instead of four zeros. Therefore, this proposed method can reduce redundancy to a certain extent. When computing the maximum threshold, the improved algorithm can initialize the maximum of every block. So, it can obviously reduce number of comparisons while scanning and coding zero trees. The coefficients in non important block will be coded in next scanning process or later, rather than be coded in the present scanning process. This method can implement the coefficients coded earlier to the non-important ones more adequately. Generally, wavelet transform coding for still image using SPIHT [12] algorithm can be modelled as Fig 7. Firstly, original image matrix goes through wavelet transform. The output wavelet coefficients are then quantized and encoded by SPIHT coder. After that, bit streams are obtained. Wavelet transform image coding using traditional SPIHT has the advantages of embedded code stream structure, high compression rate, low complexity and easy to implement [13]. However, for it, there still exist several defects. 1) When scanning the list of insignificant pixels (LIP), list of insignificant sets (LIS), or list of significant pixels (LSP), the repeated coefficient comparison can increase complexity of the algorithm.

2)The coefficients put into LIP at last scanning procedure which are smaller than the current threshold will result in redundancy.

3)Early coding for non-important coefficients in SPIHT will affect the performance of channel coding, especially unequal error protection (UEP).

Therefore, the improved SPIHT algorithm mainly makes the following changes.

*SPIHT codes four coefficients and then shifts to the next four ones. Therefore, views the four coefficients as a block [8][11]. The maximum of them regarded as the compared threshold will decrease number of comparison, which is relate with the distribution of coefficient matrix. Even more, when the maximum in the block is smaller than the current threshold or equal to it, the block will be coded with only one bit instead of four zeros. Therefore, this proposed method can reduce redundancy to a certain extent.

*When computing the maximum threshold, the improved algorithm can initialize the maximum of every block. So, it can obviously reduce number of comparison when scanning and coding zero trees. 
*The coefficients in non-important block will be coded in next scanning process or later, rather than be coded in the present scanning process [11]. This method can implement the coefficients coded earlier to the nonimportant ones more adequately.

On the basis of above-mentioned ideas for algorithm improvement, an improved algorithm is proposed and briefly describes it in the following paragraphs. In order to comprehend conveniently, symbols are given firstly. $B(i, j)$ which represents a wavelet coefficient block with coordinate $(i, j)$ includes four coefficients $(i, j)$, $(i+1, j),(i, j+l)$ and $(i+1, j+1)$, like SPIHT described in detail in and will be divided into its four off springs with coordinates $(2 i, 2 j),(2 i+2,2 j),(2 i, 2 j+2)$ and $(2 i+2,2 j+2) . O(i, j)$ : set of coordinates of all off springs of $B(i, j)$. $D(i, j)$ : set of coordinates of all descendants of $B(i, j) . L(i, j)=D(i, j)-O(i, j) . L S P=\{P(i, j) I P(i, j) E H\}$ and LIS have the same definitions as in. But the set in LIS represents either $D(i, j)$ or $L(i, j)$. To distinguish them, the type $D$ represents $D(i, j)$ and type $L$ for $L(i, j)$. Define list of insignificant block as $L I B=\{B(i, j) 1(i, j) E H\}$ instead of LIP. It stores the first coordinate of a group of $2 \mathrm{X} 2$ adjacent pixels which are regarded as a block. $\mathrm{H}$ stands for the wavelet coefficient matrix. Our algorithm encodes the sub band pixels by performing initialization and a sequence of sorting pass, refinement pass and quantization-step updating. However, differences of initialization and sorting pass still exist between the improved SPIHT [3] and traditional SPIHT.

4)Initialization: $\mathrm{LIB}=\{\mathrm{B}(\mathrm{O}, \mathrm{O}), \mathrm{B}(0,2), \mathrm{B}(2,0), \mathrm{B}(2,2)\}, \mathrm{LlS}=\{\mathrm{D}(0,2), \mathrm{D}(2,0), \mathrm{D}(2,2)\}$,

$\mathrm{T}=2 \mathrm{n}, \mathrm{Cij}$ is wavelet matrix coefficient and LSP is empty. $\mathrm{n}$ is expressed in (1).

$\mathrm{n}=|\log 2(\max (\mathrm{i}, \mathrm{j})|\mathrm{Ci}, \mathrm{j}|)|$

$\mathrm{C}_{\mathrm{i}, \mathrm{j}}$ is the matrix coefficient after DWT and $(\mathrm{i}, \mathrm{j})$ is the coordinate of $\mathrm{C}_{\mathrm{i}, \mathrm{j}}$.

5) Sorting pass: The sorting pass consists of two tests: the LIB test (LIBT) and LIS test (LIST). The LIBT will code the block or coefficients in blocks, while the LIST mainly disposes the sets in LIS. In each LIBT, if the maximum value of the coefficient block is smaller than the current threshold, the block is insignificant and ' 0 ' is the coded bit. Otherwise, 1 will be output and represent the significance of the block. Then, the four coefficients will be respectively compared to the current threshold. When the coefficient has not been put into LSP, if it is insignificant. Otherwise, 10 or 11 represent significant negative sign or significant positive sign, respectively. After that, it will be removed from the block and added to the tail of LSP. While the test is finished, the block will be removed from LIB if all the four coefficients have been put into LSP. Otherwise, the block will be tested again in next LIBT. While in LIST, the set in LIS will be tested and coded according to its type. For type D, if the maximum coefficient in $\mathrm{D}(\mathrm{i}, \mathrm{j})$ is smaller than the current threshold, the set is insignificant. Otherwise, the significant bit I will be coded and $\mathrm{D}(\mathrm{i}, \mathrm{j})$ will be divided into its children tree and four blocks with coordinate $(m, n) E Q(i, j)$ rather than four adjacent coefficients. The four blocks will be coded with the style as in LIBT, but the tail of LIB corresponding to their significances. After coding the four blocks, our algorithm will alter $D(i, j)$ to $L(i, j)$ and add $L(i, j)$ to the tail of LIS if $D(i, j)$ has grandson coefficients. Then, set $D(i, j)$ will be removed from LIS. For type $\mathrm{L}$, if the maximum coefficient in $\mathrm{L}(\mathrm{i}, \mathrm{j})$ is smaller than the current threshold, 0 will be output and represented the insignificance of the set. Otherwise, the significant bit 1 will be coded and L(i,j) will be divided into four sets $\mathrm{D}(\mathrm{m}, \mathrm{n}),(\mathrm{m}, \mathrm{n}) \mathrm{E} \mathrm{Q}(\mathrm{i}$,$) which will be added to the tail of LIS. Then, set L(i,j) will$ be removed from LIS. After completing LIPT and LIBT tests, the same refinement pass is performed and as in traditional SPIHT the threshold is updated. For the improved SPIHT, when the maximum value of a coefficient block put into LIB is small enough, only one bit will used to represent it and the four coefficients of it will not be coded until the current threshold is smaller than the maximum value. Therefore, this algorithm can better avoid repeat coding and early coding for non-important coefficients better. Moreover, this algorithm has the same scanning order and method to determine importance of wavelet coefficients as SPIHT [2][3]. Consequently, it will inherit many advantages of SPIHT.

Algorithm: ISPIHT Coding

Output: Bit stream

Input: Wavelet co-efficient or data matrix to be coded, $\mathrm{A}$, the number of threshold levels, $\mathrm{N}$.

Assign LIP $=\{\mathrm{A}(1,1), \mathrm{A}(1,2), \mathrm{A}(2,1), \mathrm{A}(2,2)\}$

Assign LIS with VTs for coordinates $(1,3),(3,1)$ and $(3,3)$ as type-0 descendent trees.

Compute Vm, M, VT(Vector Tree), Threshold (as described in the Initialization).

Assign Lp1=0;

Comment: Sorting Pass

For $\mathrm{I}=1$ to $\mathrm{N}$

Comment: LIP Testing

For each pixel in the LIP

If a pixel is significant

Send a 1 , followed by sign bit.

Delete the pixel form LIP and append its absolute value to LSP.

Else

Send a 0 
End if

End For each

Comment: LIS Testing

For each VT in LIS

If the type of VT is 0

If $\mathrm{VT}$ is significant

Send a 1

For each of the four pixels associated with the node of the VT.

If a pixel is significant,

Send a 1 followed by sign bit.

Append the absolute value of the pixel to LSP.

Else

Send a 0

Append the pixel to LIP

End If

End For each

If VT has more than 1 element

Neglect the first element, change its stype to 1 and append to LIS.

End If

Else

Send a 0

End If

Else

If VT (of type-1) is significant

Send a 1

Generate VT corresponding to the four top leftmost pixel co-ordinates of the

four $2 \times 2$ sub-matrices associated with current node.

Delete the VT from the LIS.

Else

Send a 0

End If

End If

End For each

Comment: Refinement Pass

For $\mathrm{r}=1$ to $\mathrm{Lp} 1$

If $(\operatorname{LSP}(\mathrm{r})-$ Threshold $)>=$ Threshold $/ 2$

Send a 1

Else

Send a 0

End if

End For

Lp1=No. of pixels in the LSP

Threshold=Threshold/2;

End For

The decoding algorithm is just the reverse of the coding algorithm. But a difference with coding algorithm is that the LIP and LSP are stored as co-ordinates [1] and the LIS stores only the pixel co-ordinates of the topmost modes of the descendent trees and does not store VTs. Because during the decoding, testing whether a descendent tree is significant or not requires only whether the corresponding bit is 1 or zero, it does not require exhaustive searching as in the case of coding. In other words decoding remains the same as in SPIHT except the way of representation of the tree structure.

\section{Performance Evaluation}

The Proposed algorithm was performed using MATLAB 2009a on an INTEL Pentium Core 2 duo (1.8 $\mathrm{GHz}, 2 \mathrm{G}$ RAM). We tested our algorithm on two different still images (paris city 256 x256 bbp and little river $256 \times 256$ bbp), according to a two-level wavelet decomposition using biorthogonal filters 9/7 [2, 9]. Most of the researchers have reported the performance of multispectral data in terms of bit-rate versus SNR/PSNR. Few approximated the algorithmic complexity in terms of the number of operations. Some presented a few of the original and decompressed images for visual comparison. Main purpose of all these results is to evaluate the quality of the decompressed data subjectively and objectively. However, the quantitative SNR/PSNR metrics are 
too generic because they are averaged over all the pixels. Compression efficiency is measured by the compression ratio and is estimated by the ratio of the original image size over the compressed data size. The complexity of an image compression algorithm is calculated by the number of data operations required to perform both encoding and decoding processes. Practically, it is sometimes expressed by the number of operations. For a lossy compression scheme, a distortion measurement is a criterion for determining how much information has been lost when the reconstructed image is produced from the compressed data. The most often used measurement is the mean square error (MSE). In the MSE measurement the total squared difference between the original signal and the reconstructed one is averaged over the entire signal. Mathematically,

$\mathrm{MSE}=\frac{1}{\mathrm{~N}} \sum_{\mathrm{t}=0}^{\mathrm{N}-1}\left(\hat{\mathrm{X}}_{\mathrm{i}}-\mathrm{X}_{\mathrm{i}}\right)^{2}$

Where $\hat{x}_{i}$ is the reconstructed value of $x_{i}, \mathrm{~N}$ is the number of pixels. The mean square error is commonly used because of its convenience. A measurement of MSE in decibels on a logarithmic scale is the Peak Signal-to-Noise Ratio (PSNR), which is a popular standard objective measure of the lossy codec. We use the PSNR as the objective measurement for compression algorithms throughout this paper. It is defined as follows.

$\mathrm{PSNR}=10 \log _{10}\left[255^{2} / \mathrm{MSE}\right]$

\section{Results \& Discussions}

In this paper the compression results of multispectral images with various existing techniques like DCT KLT, DWT SPIHT and proposed novel method of DWT ISPIHT are compared. Firstly the original image matrix goes through wavelet transform and the output wavelet coefficients are then quantized and encoded by ISPIHT coder. The highly scalable improved SPIHT has lot of improvements when compared to the traditional SPIHT in terms of PSNR, MSE, BER, SSIM and CR which are shown in the figures Fig 8, Fig 9, Fig 10, Fig 11 and Fig 12 respectively. The comparative results are shown in Table 1, Table 2, Table 3, Table 4 and Table 5 respectively. Analysis pertaining to the proposed method is shown in Table 6 and the overall comparative results are shown in Table 7.Thus the improved SPIHT can be implemented to multispectral images for higher image quality i.e. high PSNR without much decrease in compression ratio.

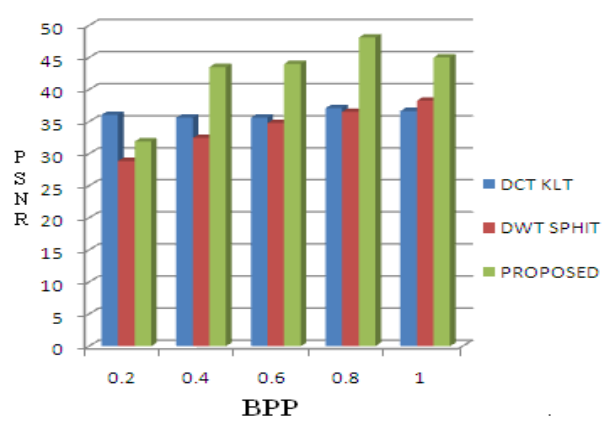

Fig 8. Graph between BPP and PSNR for various compression techniques

Table 1. PSNR values for various compression techniques

\begin{tabular}{|c|c|c|c|}
\hline BPP & DCT KLT & DWT SPHIT & PROPOSED \\
\hline 0.2 & 36.0529 & 28.8618 & 31.9182 \\
\hline 0.4 & 35.6139 & 32.4597 & 43.5163 \\
\hline 0.6 & 35.6207 & 34.7859 & 44.0182 \\
\hline 0.8 & 37.1141 & 36.5259 & 48.1372 \\
\hline 1 & 36.6576 & 38.2797 & 45.0244 \\
\hline
\end{tabular}




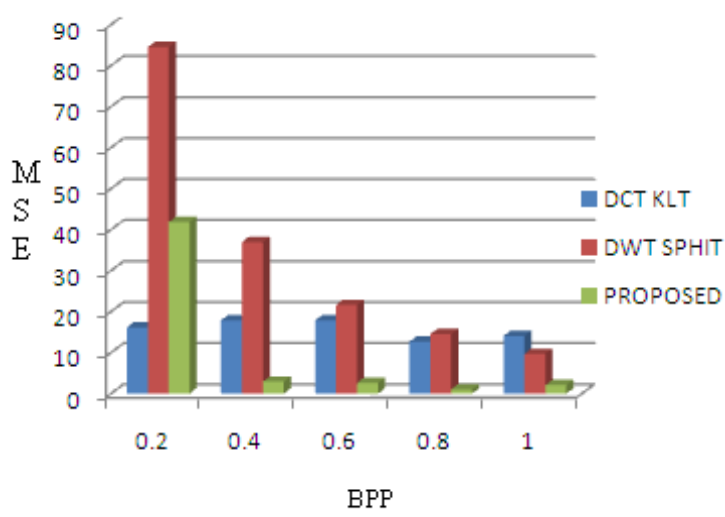

Fig 9. Graph between BPP and MSE for various compression techniques

Table 2. MSE values for various compression techniques

\begin{tabular}{|c|c|c|c|}
\hline BPP & DCT KLT & DWT SPHIT & PROPOSED \\
\hline 0.2 & 16.1358 & 84.5093 & 41.8082 \\
\hline 0.4 & 17.8521 & 36.9074 & 2.89368 \\
\hline 0.6 & 17.8241 & 21.6017 & 2.57788 \\
\hline 0.8 & 12.6379 & 14.4707 & 0.99854 \\
\hline 1 & 14.0384 & 9.66304 & 2.04475 \\
\hline
\end{tabular}

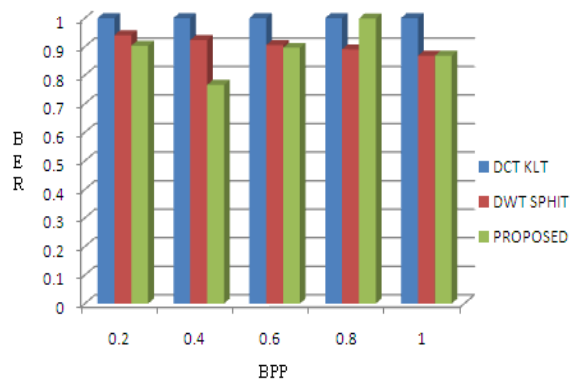

Fig 10. Graph between BPP and BER for various compression techniques

Table 3. BER values for various compression techniques

\begin{tabular}{|c|c|c|c|}
\hline BPP & DCT KLT & DWT SPHIT & PROPOSED \\
\hline 0.2 & 1 & 0.94 & 0.903152 \\
\hline 0.4 & 1 & 0.923218 & 0.766357 \\
\hline 0.6 & 1 & 0.905334 & 0.895754 \\
\hline 0.8 & 1 & 0.890442 & 0.998535 \\
\hline 1 & 1 & 0.868118 & 0.868118 \\
\hline
\end{tabular}

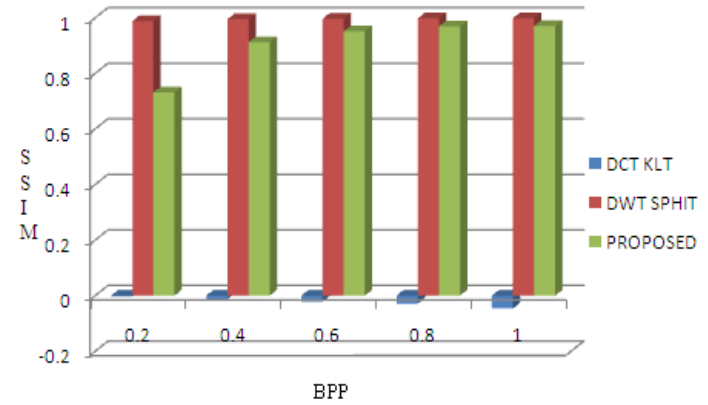

Fig 11. Graph between BPP and SSIM for various compression techniques 
Table 4. SSIM values for various compression techniques

\begin{tabular}{|c|c|c|c|}
\hline BPP & DCT KLT & $\begin{array}{c}\text { DWT } \\
\text { SPHIT }\end{array}$ & PROPOSED \\
\hline 0.2 & -0.00367 & 0.9879 & 0.73111 \\
\hline 0.4 & -0.0133 & 0.994338 & 0.91184 \\
\hline 0.6 & -0.02225 & 0.996568 & 0.95105 \\
\hline 0.8 & -0.02915 & 0.997552 & 0.96852 \\
\hline 1 & -0.04644 & 0.998387 & 0.96987 \\
\hline
\end{tabular}

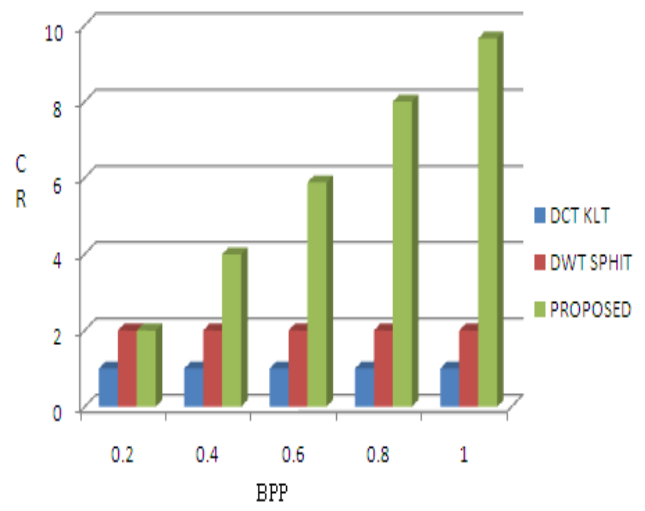

Fig 12. Graph between BPP and CR for various compression techniques

Table 5. CR values for various compression techniques

\begin{tabular}{|c|c|c|c|}
\hline BPP & DCT KLT & DWT SPHIT & $\begin{array}{c}\text { PROPOSE } \\
\text { D }\end{array}$ \\
\hline 0.2 & 1 & 2 & 2 \\
\hline 0.4 & 1 & 2 & 4 \\
\hline 0.6 & 1 & 2 & 5.88506 \\
\hline 0.8 & 1 & 2 & 8 \\
\hline 1 & 1 & 2 & 9.66038 \\
\hline
\end{tabular}

Table 6. Proposed Method Analysis

\begin{tabular}{|c|c|c|c|c|c|}
\hline $\begin{array}{c}\text { BP } \\
\text { P }\end{array}$ & PSNR & MSE & CR & BER & SSIM \\
\hline 0.2 & $\begin{array}{c}31.918 \\
2\end{array}$ & 41.8082 & 2 & $\begin{array}{c}0.90315 \\
2\end{array}$ & 0.731107 \\
\hline 0.4 & $\begin{array}{c}43.516 \\
3\end{array}$ & 2.89368 & 4 & $\begin{array}{c}0.76635 \\
7\end{array}$ & 0.911836 \\
\hline 0.6 & $\begin{array}{c}44.018 \\
2\end{array}$ & 2.57788 & $\begin{array}{c}5.88 \\
5\end{array}$ & $\begin{array}{c}0.89575 \\
4\end{array}$ & 0.951049 \\
\hline 0.8 & $\begin{array}{c}48.137 \\
2\end{array}$ & $\begin{array}{c}0.99853 \\
5\end{array}$ & 8 & $\begin{array}{c}0.99853 \\
5\end{array}$ & 0.968517 \\
\hline 1 & $\begin{array}{c}45.024 \\
4\end{array}$ & 2.04475 & $\begin{array}{c}9.66 \\
0\end{array}$ & $\begin{array}{c}0.84245 \\
6\end{array}$ & 0.969867 \\
\hline
\end{tabular}


Table 7. Comparative Results

\begin{tabular}{|c|c|c|c|c|c|c|}
\hline BPP & METHOD & PSNR & MSE & CR & BER & SSIM \\
\hline \multirow{3}{*}{0.2} & DCT KLT & 36.0529 & 16.1358 & 1 & 1 & -0.00367 \\
\hline & $\begin{array}{c}\text { DWT } \\
\text { SPHIT }\end{array}$ & 28.8618 & 84.5093 & 2 & 0.9400 & 0.9879 \\
\hline & $\begin{array}{c}\text { PROPOSE } \\
\text { D }\end{array}$ & 31.9182 & 41.8082 & 2 & 0.9031 & 0.7311 \\
\hline \multirow{3}{*}{0.4} & DCT KLT & 35.6139 & 17.8521 & 1 & 1 & -0.0132 \\
\hline & $\begin{array}{l}\text { DWT } \\
\text { SPHIT }\end{array}$ & 32.4597 & 36.9074 & 2 & 0.9232 & 0.9943 \\
\hline & $\begin{array}{c}\text { PROPOSE } \\
\text { D }\end{array}$ & 43.5163 & 2.89368 & 4 & 0.7663 & 0.9118 \\
\hline \multirow{3}{*}{0.6} & DCT KLT & 35.6207 & 17.8241 & 1 & 1 & -0.0222 \\
\hline & $\begin{array}{c}\text { DWT } \\
\text { SPHIT }\end{array}$ & 34.7859 & 21.6017 & 2 & 0.9053 & 0.9965 \\
\hline & $\begin{array}{c}\text { PROPOSE } \\
\text { D } \\
\end{array}$ & 44.0182 & 2.57788 & 5.8 & 0.8957 & 0.9510 \\
\hline \multirow{3}{*}{0.8} & DCT KLT & 37.1141 & 12.6379 & 1 & 1 & -0.0291 \\
\hline & $\begin{array}{c}\text { DWT } \\
\text { SPHIT }\end{array}$ & 36.5259 & 14.4707 & 2 & 0.8904 & 0.9975 \\
\hline & $\begin{array}{c}\text { PROPOSE } \\
\text { D }\end{array}$ & 48.1372 & 0.99853 & 8 & 0.9985 & 0.9685 \\
\hline \multirow{3}{*}{1} & DCT KLT & 36.6576 & 14.0384 & 1 & 1 & -0.0464 \\
\hline & $\begin{array}{c}\text { DWT } \\
\text { SPHIT }\end{array}$ & 38.2797 & 9.66304 & 2 & 0.8681 & 0.9983 \\
\hline & $\begin{array}{c}\text { PROPOSE } \\
\text { D }\end{array}$ & 45.0244 & 2.04475 & 9.6 & 0.8424 & 0.9698 \\
\hline
\end{tabular}

\section{Conclusions}

In this paper, we developed a compression technique for multispectral data using wavelet transform. The wavelet coefficients are using ISPIHT algorithm for optimal bit-allocation. We made an assumption that spectral correlation exists in the biorthogonal subclasses. Bands of multispectral data respond differently to DWT. The bands which are similar in Wavelet domain are encoded extremely well, this is confirmed by the results obtained using this technique. This technique is shown to work across both multispectral and hyper spectral images. We compared the numerical results obtained with our technique with some of the recently proposed techniques and got superior results. This work has shown that the compression of image can be improved by considering spectral and temporal correlations as well as spatial redundancy. The efficiency of temporal prediction was found to be highly dependent on individual image sequences. Given the results from earlier work that found temporal prediction to be more useful for image, we can conclude that the relatively poor performance of temporal prediction, for some sequences, is due to spectral prediction being more efficient than temporal. Another conclusion and future work finding from this work is that the extra compression available from image can be achieved without necessitating a large increase in decoder complexity. Indeed the presented scheme has a decoder that is less complex than many lossless image compression decoders, mainly due to the use of forward rather than backward adaptation. The results of adaptive and Non-adaptive based image compression are compared. From the results the adaptive wavelet decomposition works better than non-adaptive (Haar) wavelet decomposition. Future work aims at extending this frame work for colour images, video compressions, and de-noising applications.

\section{References}

[1]. Memon N. D., Sayood K., Magliveras S., "Lossless Compression of Multispectral Image Data," IEEE Trans. on Geosci. \& Remote Sensing, 32(2): 282 -289 (1994).

[2]. Wu X., Memon N., "Context-Based Lossless Interband Compression-Extending CALIC," IEEE Trans. on Image Processing, 9(6): $994-1001$ (2000).

[3]. Rong Zhang, Yan Qing, "A Prediction Tree-based Lossless Compression Technique of Multispectral Image Data," Journal of Remote Sensing, 2(3): 171-175 (1998).

[4]. Zheng Wu, Mingyi He, "Lossless Compression of Multispectral Imagery by Error Compensated Prediction Tree," Journal of Remote Sensing, 9(2): 143-147 (2005).

[5]. F. Rizzo, B. Carpentieri, G. Motta, and J. Storer, "Low-complexity lossless compression of hyperspectral imagery via linear prediction," IEEE Signal Process. Lett., vol. 12, no. 2, pp. 138-141, Feb. 2005.

[6]. M. Ben-Ezra, Z. C. Lin, and B. Wilburn. Penrose pixels: Superresolution in the detector layout domain. In ICCV, 2007.

[7]. H. Chang, D. Y. Yeung, and Y. Xiong. Super-resolution through neighbour embedding. In CVPR, volume 1, pages 275-282, 2004.

[8]. Yumnam Kirani Singh "ISPIHT-Improved SPIHT "A simplified and efficient subband coding scheme" Center for Development of Advanced Computing Plot: E-2/1, Block GP, Sector V, Salt Lake Electronics Complex. 
[9]. Hualiang Zhu, Chundi Xiu and Dongkai Yang “An Improved SPIHT Algorithm Based on Wavelet Coefficient Blocks for Image Coding " Beijing University of Aeronautics and Astronautics Beijing, P.R.China.

[10]. ASaid and W.APeariman, "A new fast and efficient image codec based on set partitioning in hierarchical trees," IEEE Trans. Circuits and Systems for Video Technology, vol. 6, no.3, pp.243-250, Jun.1996.

[11]. A. Said,and W. A. Pearlman, " A new, fast and

[12]. efficient image code based on set partitioning in hierarchical trees", IEEE Transactions on Circuits and Systems for Video technology, 1996, 6 (6):243-250.

[13]. F. W. Wheeler, and W. A. Pearlman, "SPIHT Image Compression without Lists," IEEE Int. Conf on Acoustics, Speech and Signal Processing(ICASSP 2000). Istanbul: IEEE, 2000 .2047-2050.

[14]. Jianxiong Wang "Study of the Image Compression based on SPIHT Algorithm" college of water resources \& hydropower and architecture, yunnan agriculture university Kunming.

\section{AUTHOR'S PROFILE}

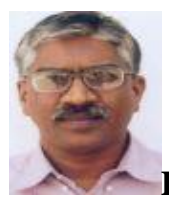

Dr. C. D. Naidu, an able administrator and accomplished educationalist, joined VNRVJIET in the year 1997 and has worked in the capacity of Vice-Principal, Dean-Academics, Professor \& Head-ECE Department and is at present Principal of the college. He was formerly associated with Vasavi

College of Engineering, Hyderabad and KSRM College of Engg.Kadapa, A. P. His research areas include Digital signal processing, Digital filters, Neural Networks, Wavelet Transforms and Image Processing and Analysis. He has published and presented several papers in International and National Conferences and Journals. He has done his Ph.D in ECE from JNTU, Hyderabad, M.Tech in Instrumentation \& Control systems from S.V.University and B.Tech in ECE from JNTU.

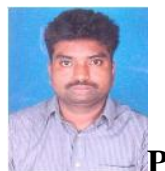

Prof. V.Bhagya Raju Completed B.E. in 2000 from Vasavi college of engineering,M.Tech in wireless\&Mobile communication from JNTUH. Presently pursuing PH.D from JNTUH in the field of compression of multispectral images and working as Prof \& HOD in ECE in Sagar Group of Institutions, SITECH, Hyderabad. He guided many research projects in M.Tech in the field of Image processing and VLSI.

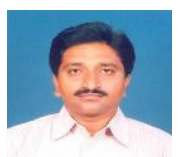

Dr. K Jaya Sankar received B.Tech degree in Electronics and Communication Engineering from NBKR Institute of Science and Technology, Vidya Nagar, and M.E \& Ph.D. degrees from the Department of ECE, OU, Hyderabad, Andhra Pradesh in 1988, 1994, and 2004 respectively. He

is working as Professor and Head of the Department of ECE at Vasavi College of Engineering, Hyderabad, India. He has 24 years of teaching experience in Digital Communication, Signal Processing, Electromagnetics, Antennas, Microwave and Radar Systems. His research areas of interest are in Coding Theory, RF \& Wireless Communications and Genetic Algorithm based antenna design. He has published more than twelve papers in national and international journals and more than twenty papers in national and international conferences. He is a Member of IEEE, Fellow of IETE and IE (I). 